Research Article

\title{
Nasal Carriage by Staphylococcus aureus among Healthcare Workers and Students Attending a University Hospital in Southern Brazil: Prevalence, Phenotypic, and Molecular Characteristics
}

\author{
Tiago Danelli $\left(\mathbb{D},{ }^{1}\right.$ Felipe Crepaldi Duarte $\left(\mathbb{D},{ }^{1}\right.$ Thilara Alessandra de Oliveira $\left(\mathbb{D},{ }^{1}\right.$ \\ Raquel Soares da Silva $\mathbb{D},{ }^{1}$ Daniela Frizon Alfieri $\mathbb{D},{ }^{2}$ Guilherme Bartolomeu Gonçalves $\mathbb{D}^{\mathrm{D}},{ }^{3}$ \\ Caio Ferreira de Oliveira $\left(\mathbb{D},{ }^{3}\right.$ Eliandro Reis Tavares $\mathbb{D}^{4},{ }^{4,5}$ Lucy Megumi Yamauchi ${ }^{(D, 3,4}$ \\ Marcia Regina Eches Perugini $\mathbb{D}^{1},{ }^{1,2}$ and Sueli Fumie Yamada-Ogatta $\mathbb{D}^{1,3,4}$ \\ ${ }^{1}$ Programa de Pós-graduação em Fisiopatologia Clínica e Laboratorial, \\ Universidade Estadual de Londrina-Rodovia Celso Garcia Cid, PR 445 km 380, Campus Universitário, Londrina, Paraná, Brazil \\ ${ }^{2}$ Departamento de Patologia, Análises Clínicas e Toxicológicas, Centro de Ciências da Saúde, Universidade Estadual de Londrina, \\ Londrina, Brazil \\ ${ }^{3}$ Programa de Pós-graduação em Microbiologia, Universidade Estadual de Londrina, Londrina, Brazil \\ ${ }^{4}$ Departamento de Microbiologia, Centro de Ciências Biológicas, Universidade Estadual de Londrina, Londrina, Brazil \\ ${ }^{5}$ Programa Nacional de Pós-Doutorado-CAPES, Universidade Estadual de Londrina, Londrina, Brazil
}

Correspondence should be addressed to Sueli Fumie Yamada-Ogatta; ogatta@uel.br

Received 7 July 2020; Accepted 17 November 2020; Published 4 December 2020

Academic Editor: Massimiliano Lanzafame

Copyright ( 12020 Tiago Danelli et al. This is an open access article distributed under the Creative Commons Attribution License, which permits unrestricted use, distribution, and reproduction in any medium, provided the original work is properly cited.

\begin{abstract}
Background. Staphylococcus aureus can asymptomatically colonize the human anterior nares and skin, and nasal colonization by this bacterium represents a potential risk for development of invasive infections. The aim of this study was to determine the prevalence of $S$. aureus nasal carriage among healthcare workers and students attending a university hospital and to characterize the isolates phenotypically and molecularly. Methods. A cross-sectional study was performed with 324 volunteers. Cultures from nasal samples were obtained and S. aureus isolates were characterized according to their antimicrobial susceptibility profile and four virulence factors-encoding genes. MRSA isolates were characterized regarding their oxacillin/cefoxitin susceptibility, SCCmec, and REP-PCR types. Potential risks for S. aureus and MRSA carriage were analyzed. Results. Of 324 nasal samples, $42.9 \%$ were identified as S. aureus, of which $28.8 \%$ were MRSA. S. aureus carriers were significantly higher in males and students $(\mathrm{OR}=2.898,95 \% \mathrm{CI} 1.553-5.410)$; however, no variables were associated with MRSA carriage. All isolates were susceptible to vancomycin and the highest rate of resistance was observed for penicillin (90.6\%). All isolates harbored the coa gene, and $97.8 \%$, the $i c a A$ gene; $15.8 \%$ and $6.5 \%$ were positive for $t s t$ and $l u k S-P V / l u k F-P V$ genes, respectively. Among MRSA isolates, $45 \%$ carried the mecA gene but were phenotypically susceptible to oxacillin/cefoxitin; two harbored the tst and none had $l u k S-P V / l u k F-P V$ genes. All MRSAs were distributed into six SCCmec types and type I (62.5\%) was the most frequent. REP-PCR typing identified four main clusters among MRSA isolates. Conclusion. High prevalence of healthcare workers and students were identified as nasal carriers of $S$. aureus exhibiting different antimicrobial resistance profiles, including mecA-positive oxacillin-susceptible $S$. aureus (OS-MRSA) and the presence of virulence-encoding genes. Both cohorts may represent potential sources for the emergence of a successful S. aureus strain highly adapted to the hospital environment.
\end{abstract}

\section{Introduction}

Staphylococcus aureus can interact with its host as a commensal member of the microbiota [1] or as an opportunistic pathogen causing a wide range of community and hospitalassociated infections [2-7]. In humans, this bacterium can colonize multiple body sites, but the nose (anterior nares) is the frequent ecological niche of $S$. aureus carriage. Indeed, 
around $20-30 \%$ of the human population can harbor persistently and asymptomatically this bacterium in this niche [1]. Importantly, persistent nasal colonization by $S$. aureus may increase the risk for subsequent infections and this scenario is even more complicated in hospitalized and immunocompromised individuals who can develop invasive infections with high morbidity and mortality rates $[8,9]$.

Nasal colonization by $S$. aureus may begin within the first days of human life and the horizontal transmission from colonized mother seems to be the major source of $S$. aureus carriage in newborns [10]. After birth, hands are the main vector for transmitting $S$. aureus from the surfaces to the nose $[11,12]$. Particularly in hospitals, healthcare workers and patients who are nasal carriers may be the source for the transmission and spread of $S$. aureus in these settings [13-18]. Similarly, students who have continuous hospital exposure during their internship may represent an important source for transmitting $S$. aureus within the hospital environments $[14,19]$. Crucially, high rates of colonization by methicillin-resistant $S$. aureus (MRSA) have been reported among healthcare workers [16, 17], and increasing rates of MRSA colonization among students with continuous hospital exposure have also been reported [19]. Besides their role in S. aureus transmission in hospital settings, healthcare workers and students may also serve as the vector for cross-transmission of MRSA between the community and hospital populations $[14,18]$.

Altogether these data indicate the importance of screening $S$. aureus nasal carriers in both healthcare workers and students as a component of the control of $S$. aureus infections in hospitals. In this regard, few studies on the prevalence of $S$. aureus colonization in healthcare workers and students attending the Brazilian hospital environments were reported in the literature.

The University Hospital of Universidade Estadual de Londrina (UH-UEL) is a public teaching hospital and the reference in high complexity cases in the north of Paraná, Brazil. The hospital serves as an internship field for undergraduate students of Medicine, Pharmacy, Nursing, and Physiotherapy and offers postgraduate programs in different biomedical skills. In a retrospective study performed during 2001-2015, MRSA accounted for $43.7 \%$ of bacteremia in hospitalized patients [5]. However, no study on nasal colonization by $S$. aureus of healthcare workers and students attending the hospital was performed so far. Therefore, the aims of this study were to estimate the prevalence of and the potential risk for $S$. aureus nasal carriage among healthcare workers and students of the UH-UEL and to characterize the isolates by phenotypic and molecular methods.

\section{Materials and Methods}

2.1. Study Design. A cross-sectional study was carried out with the community of the UH-UEL from December 2017 to May 2018. The sample size was calculated using the software EPI Info ${ }^{\mathrm{TM}} 7$ (Atlanta, USA) and was based on the population (students and professionals) attending the UH-UEL, which amounts about 2000 individuals during the period of the study. Assuming a prevalence of $S$. aureus colonization of about $40 \%$ [20] and a confidence interval of 95\%, at least 324 individuals were included in this study. The collection of data occurred at the time of nasal swab sampling through a standardized questionnaire that contained demographic variables, antibiotic use and/or hospitalization in the last three months, and direct contact with hospitalized patients. The study protocol was approved by the Ethics Committee of the UEL (CAAE 79663417.2.0000.5231-opinion number 2.421.361-CEP-UEL).

2.2. Collection and Processing of Nasal Swabs. One nasal sample was collected from each participant using the Stuart collection device (COPAN Diagnostic, Italy). The swab was introduced into a nostril and gently rotated three times, transported to the laboratory, and processed up to $2 \mathrm{~h}$ after collection. Nasal samples were inoculated into Tryptone Soya Broth (TSB, Oxoid, Brazil) supplemented with $6.5 \%$ sodium chloride and incubated at $35^{\circ} \mathrm{C}$ for $24 \mathrm{~h}$. After, the sample was subcultured on Mannitol Salt Agar (Oxoid, Brazil) at $35^{\circ} \mathrm{C}$ for $24 \mathrm{~h}$. Suggestive colonies of staphylococci were subjected to standard phenotypic identification [21]. Species identification was confirmed by a multiplex-PCR targeting the nuc gene (encoding thermonuclease) [22]. Bacteria were stored at $-80^{\circ} \mathrm{C}$ in $\mathrm{TSB}$ containing $30 \%$ glycerol.

2.3. Antimicrobial Susceptibility Testing. The antibacterial susceptibility profiles for 12 antimicrobials were evaluated by the disk diffusion method. The minimal inhibitory concentration (MIC) for oxacillin was determined by the broth microdilution assay. The inducible clindamycin resistance was determined by the double disk diffusion method. All methods were performed as recommended by the Clinical and Laboratory Standards Institute [23]. The susceptibility breakpoints were those recommended by CLSI [23] except tigecycline that was interpreted according to the European Committee on Antimicrobial Susceptibility Testing [24]. Enterococcus faecalis ATCC 29212 and S. aureus ATCC 25923 were used as quality controls. Cefoxitin disk and oxacillin MIC were used to define MRSA.

To detect heterogeneous vancomycin-intermediate S. aureus (hVISA), $10 \mu \mathrm{L}$ of bacterial suspension $\left(1.0-2.0 \times 10^{8} \mathrm{CFU} / \mathrm{mL}\right)$ was spotted in duplicate onto Brain Heart Infusion agar supplemented with $3 \mu \mathrm{g} / \mathrm{mL}$ and $4 \mu \mathrm{g} /$ $\mathrm{mL}$ vancomycin [25]. The systems were incubated at $35^{\circ} \mathrm{C}$ and the results were recorded at 24 and $48 \mathrm{~h}$ of incubation. S. aureus ATCC 29213 (MSSA), S. aureus BEC 9393 (MRSA), S. aureus $\mathrm{Mu}_{3}$ ATCC 700698 (hVISA), S. aureus $\mathrm{Mu}_{50}$ ATCC 700699 (VISA), and E. faecalis ATCC 51299 (VRE) were included as quality controls.

2.4. DNA Extraction. A single colony of each bacterium was cultured in $3 \mathrm{~mL}$ TSB at $35^{\circ} \mathrm{C}$ for $24 \mathrm{~h}$. The cells were harvested by centrifugation $(10,000 \times \mathrm{g}$ for $5 \mathrm{~min})$, washed once with sterile $0.15 \mathrm{M}$ phosphate-buffered saline $\mathrm{pH} 7.2$, and resuspended in $300 \mu \mathrm{L}$ sterile solution $(10 \mathrm{mM}$ Tris- $\mathrm{HCl}$, $1 \mathrm{mM}$ EDTA pH8.0, and $1.0 \mathrm{mg} / \mathrm{mL}$ lysozyme). Genomic 
DNA was extracted as described previously [26], and $2 \mu \mathrm{L}$ were used in all amplification reactions.

\subsection{Detection of Genes Encoding mecA and Virulence Factors} by PCR. The gene mecA (encoding PBP2a) was detected as described by Milheiriço et al. [27]; the genes icaA (encoding $N$-acetylglucosaminyltransferase of the intercellular adhesion locus), lukS-PV/lukF-PV (encoding Panton-Valentine leukocidin-PVL), and tst (encoding toxic shock syndrome toxin-TSST-1) were detected as described by Campbell et al. [28], and the gene coa was detected as described by Tiwari et al. [29]. S. aureus BEC $9393\left(\mathrm{mecA}^{+}, i c a A^{+}, t s t^{+}\right)$and S. aureus ATCC $25923\left(c o a^{+}, l u k S-P V^{+} / l u k F-P V^{+}\right)$were used as controls.

2.6. MRSA Typing. SCCmec typing was performed by multiplex-PCR assay as described by Milheiriço et al. [27]. Nontypeable isolates were designated NT. S. aureus NCTC10442 (type I), N315 (type II), 85/2082 (type III), 81/ 108 (type IV), WIS [WBG8318] (type V), and HDE288 (type VI) strains were used as controls.

The genetic relatedness of all MRSA was analyzed by repetitive element sequence based-PCR (REP-PCR) [30]. Fingerprintings that had more than one band differing in size were considered different REP-PCR types [31]. Banding patterns were categorized using the UPGMA algorithm and Jaccard coefficient [32] of the Bionumerics v.6.5 software (Applied Mathematics, Kortrijk, Belgium), with the band tolerance set at $3 \%$ and the threshold cutoff value set at $85 \%$ [3].

2.7. Statistical Analyses. Analyses of contingency tables $\left(\chi^{2}\right.$ test) were employed to check the associations between categorical variables and diagnostic groups. The Kolmogorov-Smirnov test was used to assess the normality of distribution of age. Categorical variables were expressed as absolute number $(n)$ and percentage (\%) and continuous variables were expressed as mean \pm standard deviation. The association between explanatory variables and colonization was evaluated using three different models of automatic stepwise binary logistic regression analysis controlled for covariates that may confound the association of interest. All statistical analyses were performed using IBM SPSS windows version 20. Tests were 2-tailed and an alpha level of 0.05 indicated statistically significant results.

\section{Results and Discussion}

3.1. Baseline Characteristics of Participants and Prevalence of Nasal Carriage with S. aureus and MRSA. A total of 324 volunteers were enrolled in this study, where $103(31.8 \%)$ were healthcare workers, 128 (39.5\%) undergraduate, and 93 (28.7\%) postgraduate students. The mean age of all participants was $30 \pm 11.2$ years ranging from 19 to 73 years, where $88(27.2 \%)$ were males and $236(72.8 \%)$ were females.

Although one single nasal sampling was performed on each participant, which does not allow the identification of persistent carriers [33], a high prevalence of $S$. aureus carriers was detected in this study. The overall prevalence of S. aureus nasal carriage was $42.9 \%$ (139/324); among them, $28.8 \%$ (40/139) were identified as MRSA carriers; that is, the isolate harbored the mecA gene, representing $12.3 \%$ of all participants (Figure 1).

3.2. S. aureus Nasal Carriage Was Significantly Higher in Males and Students. Colonization by S. aureus depends on bacterial ability to survive and adapt in the host's niches. These include overcoming innate and adaptive defenses of the host along with coexisting with other members of the microbiota that may promote or inhibit its growth [1]. Moreover, several factors including younger age, being male, contact with healthcare workers, professional occupation, and geographical region have been correlated with $S$. aureus nasal colonization [13, 34-36].

In this study, we analyzed six descriptive characteristics of the participants (gender, age, occupation, antibiotic use and/or hospitalization in the last three months, and direct contact with hospitalized patients) and their potential risk for $S$. aureus nasal carriage (Table 1). In order to evaluate the independence of associations, we have carried out three different automatic stepwise logistic regression analyses. In model \#1 we added gender and occupation, both variables were positively associated with colonization with $S$. aureus ( $p=0.004$ and $p=0.019$, respectively) $\left(\chi^{2}=12.337, \mathrm{~d} f=2\right.$, $p=0.002)$. The second model (\#2) is the model \#1 added to the other explanatory variables evaluated in this study. Gender (male) remained associated with colonization $(p=0.003)$, whereas age was negatively associated with colonization $(p=0.021)(\chi 2=12.915, \mathrm{~d} f=2, p=0.002)$. Finally, in model \#3, when evaluating the interaction between gender (male) and occupation (student), only students of male gender were independently associated with colonization by $S$. aureus $(p=0.001)(\chi 2=1.733, \mathrm{~d} f=1$, $p=0.001)$. Furthermore, model \#3 contributed to correctly assessing $61.8 \%$ of the cases, with high sensitivity (90.2\%); however, it presented low specificity (23.9\%). The other explanatory variables were not associated with $S$. aureus nasal carriage $(p>0.05)$ (Table 2$)$. Accordingly, male participants had 2.107 (95\% CI: $1.268-3.502 ; p=0.004)$ times the chance of being a carrier of $S$. aureus compared to female participants. Additionally, students had 1.819 (95\% CI: $1.105-2.996, p=0.019)$ times the chance of being a carrier of S. aureus compared to healthcare workers (Table 2). None of the analyzed variables were associated with MRSA nasal carriage (Table 1).

Few studies analyzing together both cohorts and healthcare workers and students attending a hospital are described in the literature. Hogan et al. [14] reported a low prevalence of $S$. aureus nasal colonization in healthcare workers $(10.4 \%)$ and in nonmedical students $(11.4 \%)$ of the University of Madagascar. In contrast to our results, the carriage was higher in female participants and increased with age. Hussein et al. [15] reported a prevalence of $22.5 \%$ and $18.7 \%$ of S. aureus nasal carriage in healthcare workers, and students of a preparatory school that had no contact 


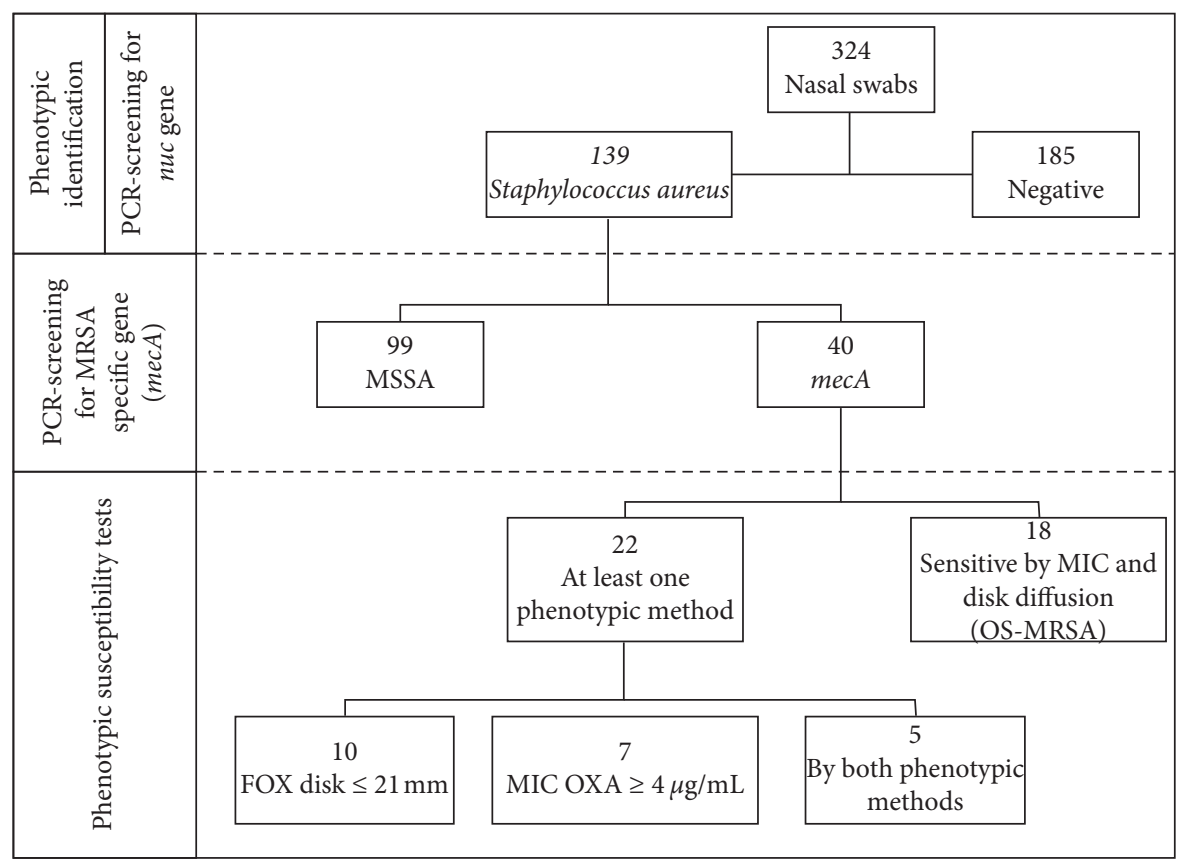

FIgURE 1: Flowchart of the procedures and the results for nasal swabs obtained from healthcare workers and students attending the University Hospital of Londrina from December 2017 to May 2018. PCR: polymerase-chain reaction; MSSA: methicillin-susceptible Staphylococcus aureus; MRSA: methicillin-resistant S. aureus; MIC: minimal inhibitory concentration; OS-MRSA: mecA-positive oxacillinsusceptible S. aureus; FOX: cefoxitin; OXA: oxacillin.

TABle 1: Potential risk factors for nasal carriage with Staphylococcus aureus and methicillin-resistant S. aureus (mecA-positive) isolates among healthcare workers and students attending the University Hospital of Londrina from December 2017 to May 2018.

\begin{tabular}{|c|c|c|c|c|c|c|c|}
\hline \multirow{2}{*}{\multicolumn{2}{|c|}{ Variable }} & \multicolumn{3}{|c|}{ Staphylococcus aureus } & \multicolumn{3}{|c|}{ mecA } \\
\hline & & Negative $n(\%)$ & Positive $n(\%)$ & $p$ value & Negative $n(\%)$ & Positive $n(\%)$ & $p$ value \\
\hline \multirow{2}{*}{ Gender } & Male & $40(21.6)$ & $48(34.5)$ & 0.010 & $32(32.3)$ & $16(40.0)$ & 0.389 \\
\hline & Female & $145(78.4)$ & $91(65.5)$ & & $67(67.7)$ & $24(60.0)$ & \\
\hline Age (years) & & $31.2(11.9)$ & $28.8(10.8)$ & 0.054 & $28.6(10.2)$ & $29.3(9.5)$ & 0.719 \\
\hline \multirow{2}{*}{ Antibiotic use } & No & $151(81.6)$ & $115(82.7)$ & 0.796 & $79(79.8)$ & $36(90.0)$ & 0.150 \\
\hline & Yes & $34(18.4)$ & $24(17.3)$ & & $20(20.2)$ & $4(10.0)$ & \\
\hline \multirow{2}{*}{ Hospitalization } & No & $176(95.1)$ & $137(98.6)$ & 0.092 & $97(98.0)$ & $40(100)$ & 0.365 \\
\hline & Yes & $9(4.9)$ & $2(1.4)$ & & $2(2.0)$ & $0(0)$ & \\
\hline \multirow{2}{*}{ Occupation } & Student & $118(63.8)$ & $103(74.1)$ & 0.048 & $76(76.8)$ & $27(67.5)$ & 0.259 \\
\hline & Professional & $67(36.2)$ & $36(25.9)$ & & $23(23.2)$ & $13(32.5)$ & \\
\hline \multirow{5}{*}{ Student } & Nursing & $24(20.3)$ & $14(13.6)$ & 0.596 & $11(14.5)$ & $3(11.1)$ & 0.698 \\
\hline & Pharmacy & $19(16.1)$ & $20(19.4)$ & & $17(22.4)$ & $3(11.1)$ & \\
\hline & Physiotherapy & $10(8.5)$ & $6(5.8)$ & & $4(5.3)$ & $2(7.4)$ & \\
\hline & Medicine & $17(14.4)$ & $18(17.5)$ & & $13(17.1)$ & $5(18.5)$ & \\
\hline & Postgraduate & $48(40.7)$ & $45(43.7)$ & & $31(40.8)$ & $14(51.9)$ & \\
\hline \multirow{2}{*}{ Patient contact } & No & $51(27.6)$ & $28(20.1)$ & 0.124 & $23(23.2)$ & $5(12.5)$ & 0.153 \\
\hline & Yes & $134(72.4)$ & $111(79.9)$ & & $76(76.8)$ & $35(87.5)$ & \\
\hline
\end{tabular}

The continuous variables were expressed as mean and standard deviation (SD) and analyzed with Student's $t$-test; the categorical variables were expressed as number $(n)$ and percentage and analyzed with $\chi^{2}$ or Fisher exact test.

with a hospital, respectively, in Iraq. None of the variables analyzed (gender, age, and working hours per day or year) were associated with S. aureus colonization. Finally, ElMahdy et al. [18] reported a prevalence of $26.0 \%$ of S. aureus nasal carriers among both healthcare workers and clinical students in Saudi Arabia. These authors did not evaluate the risk factors associated with colonization.

Previous studies performed with individual cohorts showed that the prevalence of nasal colonization by
S. aureus and MRSA is highly variable. For instance, reported prevalence of $S$. aureus and MRSA carriers ranged from 12.0 to $39.8 \%$ and 0.2 to $22.6 \%$, respectively, among healthcare workers $[13,16,17,37,38]$, and 15.0 to $40.8 \%$ and 0 to $8.0 \%$, respectively, among students $[20,39-41]$.

In Brazil, few reports about the prevalence of nasal S. aureus carriage in healthcare workers and students have been reported. In a study conducted with healthcare workers 
TABLE 2: Bivariate logistic regression analyses of descriptive variables associated with Staphylococcus aureus nasal carriage among healthcare workers and students attending the University Hospital of Londrina from December 2017 to May 2018.

\begin{tabular}{|c|c|c|c|}
\hline \multirow{2}{*}{ Model } & \multirow{2}{*}{ Explanatory variable } & \multicolumn{2}{|c|}{ Staphylococcus aureus } \\
\hline & & $p$ value & OR $(95 \% \mathrm{CI})$ \\
\hline \multirow{2}{*}{$\# 1$} & Gender (male) & 0.004 & $2.107(1.268-3.502)$ \\
\hline & Occupation (student) & 0.019 & $1.819(1.105-2.996)$ \\
\hline \multirow{6}{*}{$\# 2$} & Gender (male) & 0.003 & $2.182(1.309-3.665)$ \\
\hline & Age & 0.021 & $0.975(0.955-0.996)$ \\
\hline & Occupation (student) & 0.537 & - \\
\hline & Antibiotic use & 0.850 & - \\
\hline & Hospitalization & 0.251 & - \\
\hline & Patient contact & 0.147 & - \\
\hline \multirow{5}{*}{$\# 3$} & Gender (male) occupation (student) & 0.001 & $2.898(1.553-5.410)$ \\
\hline & Age & 0.218 & - \\
\hline & Antibiotic use & 0.961 & - \\
\hline & Hospitalization & 0.215 & - \\
\hline & Patient contact & 0.145 & - \\
\hline
\end{tabular}

OR: odds ratio; CI: confidence interval.

of the University Hospital of Recife, Pernambuco, a prevalence of $25.7 \%(52 / 202)$ of $S$. aureus carriers was identified, where $5.8 \%(3 / 52)$ were MRSA [13]. In another study, a prevalence of $21.7 \%(30 / 138)$ was observed in nursing students of the Federal University of Piauí, Piauí, and, among the carriers, $23.3 \%$ (7/30) were colonized with MRSA [41]. Similar to our results, a high prevalence (102/250, $40.8 \%$ ) of colonization by $S$. aureus was found among undergraduate biomedical students of the State University of Maringá, in the northwest of Paraná. MRSA was identified in $5.9 \%(6 / 102)$ of colonized students [20].

\subsection{S. aureus Isolated from Healthcare Workers and Students} Exhibits Different Antimicrobial Susceptibility Patterns. In our hospital, vancomycin and linezolid are the most used antimicrobials for the treatment of MRSA infections, whereas the others analyzed in this study are used in MSSA infections. The majority of $S$. aureus isolates exhibited susceptibility to most antimicrobials, and this result is consistent with studies reported previously [14], including in Brazil [13, 20, 41]. According to the phenotypic results, six (4.3\%) out of 139 isolates were susceptible to all antibacterial agents tested in this study, and all were susceptible to vancomycin. The majority of the isolates were also susceptible to chloramphenicol $(135 / 139,97.1 \%)$, ciprofloxacin $(120 / 139,86.3 \%)$, gentamicin $(124 / 139,89.2 \%)$, linezolid $(138 / 139,99.3 \%)$, rifampicin $(138 / 139,99.3 \%)$, sulfamethoxazole/trimethoprim $(123 / 139,88.5 \%)$, tetracycline $(129 /$ $139,92.8 \%)$, and tigecycline (138/139, 99.3\%). Conversely, a high rate of resistance was observed for penicillin (126/139, $90.6 \%)$, erythromycin $(93 / 139,66.9 \%)$, and clindamycin (82/ $139,59.0 \%$ ) (Table 3). Among erythromycin-resistant isolates, $82.8 \%$ (77/93), 3.2\% (3/93), and 14.0\% (13/93) displayed the $\mathrm{iMLS}_{\mathrm{B}}, \mathrm{CMLS}_{\mathrm{B}}$, and $\mathrm{M}$ phenotypes, respectively.

Similar to our results, high resistance rates to beta-lactams antimicrobials, such as ampicillin and penicillin, have been reported to $S$. aureus isolated from nasally colonized individuals from both cohorts $[17,18,20,36,40,41]$.
However, the rates of clindamycin/erythromycin resistance may vary according to the geographical region. Low resistance rates were observed in studies from Colombia [39] and Italy [40]. Conversely and consistent with our findings, high rates of resistance to both antimicrobials were detected in Iran [38], China [36], and Brazil [20, 41]. Finally, in some countries, high resistance rates were detected only for erythromycin, including Saudi Arabia [18] and Ethiopia [17].

Herein, S. aureus isolates were classified into 38 groups according to the antimicrobial resistance profile (antibiotype) (Table 4). Although a high number of antibiotypes was detected, the majority consisted of a single isolate, and the most frequent profile was coresistance to penicillin, erythromycin, and clindamycin (43/139, 30.9\%), followed by resistance to penicillin $(30 / 139,21.6 \%)$. Importantly, $24.5 \%$ (34/139) of these isolates were classified as multidrug-resistant, that is, being resistant to three or more antimicrobial classes [42].

Another important finding of our study is that a high proportion of MRSA isolates $(18 / 40,45.0 \%)$ was not detected by phenotypic methods used for identifying methicillin resistance. Indeed, MRSA isolates $(n=40)$ exhibited different patterns of susceptibility to cefoxitin/oxacillin: (a) 22 (55.0\%) exhibited methicillin-resistant phenotype in at least one test; (b) 15 (37.5\%) were classified as resistant according to $30 \mu \mathrm{g}$ cefoxitin disk diffusion assay; (c) 12 (30.0\%) displayed oxacillin MIC values $\geq 4 \mu \mathrm{g} / \mathrm{mL}$ and were classified as resistant; (d) only five isolates exhibited concordant results in both phenotypic methods; (e) 18 (45.0\%) were susceptible to methicillin by both phenotypic methods and can be classified as mecA-positive oxacillin-susceptible $S$. aureus (OS-MRSA) (Figures 1 and 2).

Currently, OS-MRSA isolated from both infection $[7,43,44]$ and nasal colonization [18] has been increasingly reported worldwide. The presence of OS-MRSA carriers and the potential risk of its transmission within the hospital and community are of concern. This bacterium can be misidentified as MSSA by phenotypic methods routinely used in 
TABLE 3: Antimicrobial susceptibility of 139 Staphylococcus aureus isolated from nasal swab of healthcare workers and students attending the University Hospital of Londrina in southern Brazil.

\begin{tabular}{|c|c|c|c|c|c|c|}
\hline \multirow{2}{*}{ Antimicrobial } & \multicolumn{3}{|c|}{ MSSA $n=99(\%)$} & \multicolumn{3}{|c|}{ MRSA $n=40(\%)$} \\
\hline & S & I & $\mathrm{R}$ & S & I & $\mathrm{R}$ \\
\hline Penicillin & $13(13.1)$ & - & $86(86.9)$ & - & - & $40(100.0)$ \\
\hline Cefoxitin & $99(100.0)$ & - & - & $25(62.5)$ & - & $15(37.5)$ \\
\hline Oxacillin & $99(100.0)$ & - & - & $28(70.0)$ & - & $12(30.0)$ \\
\hline Chloramphenicol & $97(98.0)$ & - & $2(2.0)$ & $38(95.0)$ & $1(2.5)$ & $1(2.5)$ \\
\hline Ciprofloxacin & $90(90.9)$ & $3(3.0)$ & $6(6.1)$ & $30(75.0)$ & $6(15.0)$ & $4(10.0)$ \\
\hline Clindamycin & $47(47.5)$ & $2(2.0)$ & $50(50.5)$ & $8(30.0)$ & & $32(80.0)$ \\
\hline Erythromycin & $34(34.3)$ & $7(7.1)$ & $58(58.6)$ & $4(10.0)$ & $1(2.5)$ & $35(87.5)$ \\
\hline Gentamicin & $91(91.9)$ & $1(1.0)$ & $7(7.1)$ & $33(82.5)$ & $2(5.0)$ & $5(12.5)$ \\
\hline Linezolid & $99(100.0)$ & - & - & $39(97.5)$ & - & $1(2.5)$ \\
\hline Rifampicin & $98(99.0)$ & - & $1(1.0)$ & $40(100.0)$ & - & - \\
\hline Sulfamethoxazole/Trimethoprim & $87(87.9)$ & $1(1.0)$ & $11(11.1)$ & $36(90.0)$ & $1(2.5)$ & $3(7.5)$ \\
\hline Tetracycline & $93(93.9)$ & $1(1.0)$ & $5(5.1)$ & $36(90.0)$ & $1(2.5)$ & $3(7.5)$ \\
\hline Tigecycline & $98(99.0)$ & - & $1(1.0)$ & $40(100.0)$ & - & - \\
\hline Vancomycin & $99(100.0)$ & - & & $40(100.0)$ & - & - \\
\hline
\end{tabular}

Antimicrobial susceptibility was determined by disk diffusion, except oxacillin and vancomycin that were determined by broth microdilution assay [23] and the agar-screen test [25], respectively. Disk diffusion and microdilution results were interpreted as recommended by CLSI [23] except tigecycline that was interpreted according to the EUCAST [24]. —: not detected; S: susceptible; I: intermediate; R: resistant.

most clinical laboratories, including ours, posing a threat to the treatment of staphylococcal diseases. Indeed, we recently reported the first case of fatal infection caused by an OSMRSA SCCmec type IV that was identified as oxacillinsusceptible by the automated phenotypic method and Etest $(\mathrm{MIC}=0.75 \mu \mathrm{g} / \mathrm{mL})$ in our hospital [7]. Altogether, these findings corroborate the need for additional tests to accurately distinguish the various methicillin-susceptibility phenotypes of $S$. aureus.

\subsection{MSSA Isolates Exhibit Higher Virulence Potential than} MRSA Isolates. The overall prevalence of genes encoding virulence factors in all S. aureus was as follows: coa, 100\% (139/ 139); icaA, 97.8\% (136/139); tst, 15.8\% (22/139); and lukS-PVI lukF-PV, 6.5\% (9/139). Most isolates (109/139, 78.4\%) harbored the coa and icaA genes; the other isolates harbored the following combination: coa, icaA, tst, (18/139, 12.9\%); coa, icaA, lukS-PV/lukF-PV, (8/139, 5.8\%); coa, tst, (2/139, 1.4\%); coa, icaA, tst, lukS-PV/lukF-PV, (1/139, 0.7\%) (Table 5).

Not surprisingly, all $S$. aureus harbored the coa gene that encodes coagulase, a secreted factor that induces intravascular coagulation, allowing bacterial aggregation in blood and hence promotes its survival and dissemination [45]. Moreover, coagulase activity has been used to differentiate between S. aureus (coagulase positive) and those staphylococci historically classified as less pathogenic (coagulase negative) [46]. The gene $i c a A$ is a component of the icaABDC operon that encodes the enzymes involved in the biosynthesis of polysaccharide intercellular adhesin (PIA). This adhesin promotes intercellular aggregation during the maturation stage of PIA-dependent biofilm formation by $S$. aureus [47] and is essential for bacterial virulence in murine model of systemic infection [48].

Notably, the genes lukS-PV/lukF-PV and tst were, respectively, detected exclusively and predominantly in MSSA isolates of our study, and, in general, these isolates exhibited resistance to fewer antimicrobials. Accordingly, the lukS$P V / l u k F-P V$-positive isolates displayed the antibiotypes 3, 6, 7,12 , and 14; two tst-positive isolates were susceptible to all antimicrobials and the others displayed antibiotypes 1, 3, 6, $8,17,20$, and 23 . Only the antibiotic use was positively associated with $S$. aureus harboring lukS-PV/lukF-PV genes (44.4\% positive versus $15.4 \%$ negative) (OR: $4.400,95 \% \mathrm{CI}$ : $1.087-17.815, p=0.038)$ and this result remained associated after correction for the other descriptive variables (OR: 4.360, 95\% CI: $1.077-17.655, p=0.039$ ). No variables were associated with the $t$ st gene (data not shown).

The presence of PVL encoding genes is highly variable and can be related to $S$. aureus strain types. Indeed, PVL encoding genes have been primarily associated with community-acquired methicillin-resistant S. aureus (CA-MRSA) [49]. On the other hand, tst gene has been frequently associated with MSSA strains [50]. However, both genes have also been detected in $S$. aureus exhibiting different characteristics than those of these strains [3, 6, 14, 39, 49].

PVL, a bicomponent $\beta$-pore-forming toxin, targets the cell membranes of polymorphonuclear leukocytes, monocytes, and macrophages and causes tissue necrosis [51]. Invasive infections caused by PVL-positive $S$. aureus strains have been associated with poor outcome and high mortality rates, regardless of the methicillin-resistance $[52,53]$. Importantly, the PVL encoding genes are carried by lysogenic bacteriophages that can be induced by the treatment with various antibacterial agents, including ciprofloxacin, imipenem, trimethoprim and/or sulfamethoxazole, and tobramycin [49], thus, facilitating the horizontal transmission among different isolates [51]. Furthermore, it has been known that sub-MIC of beta-lactam antibiotics, which can be found within biofilms, enhances the production of PVL in vitro and in vivo [54]. Although the clinical significance of this effect is still unknown, these data highlight the importance of the 
TABLE 4: Distribution of antimicrobial resistance profiles of methicillin-susceptible and methicillin-resistant Staphylococcus aureus isolated from the anterior nares of healthcare workers and students attending the University Hospital of Londrina in southern Brazil.

\begin{tabular}{|c|c|c|c|c|}
\hline \multirow{2}{*}{ Group } & \multirow{2}{*}{ Antimicrobial resistance profile } & \multicolumn{3}{|c|}{ Number of isolates } \\
\hline & & MSSA, $n=93$ & MRSA, $n=40$ & Total, ${ }^{*} N=139(\%)$ \\
\hline 1 & STX & 1 & - & $1(0.7)$ \\
\hline 2 & $\mathrm{TE}$ & 1 & - & $1(0.7)$ \\
\hline 3 & $\mathrm{P}$ & 27 & 3 & $30(21.6)$ \\
\hline 4 & $\mathrm{P}, \mathrm{CLO}$ & 1 & - & $1(0.7)$ \\
\hline 5 & $\mathrm{P}, \mathrm{CN}$ & 1 & - & $1(0.7)$ \\
\hline 6 & P, STX & 2 & - & $2(1.4)$ \\
\hline 7 & $\mathrm{P}, \mathrm{E}$ & 5 & 1 & $6(4.3)$ \\
\hline 8 & $\mathrm{P}, \mathrm{DA}$ & 1 & - & $1(0.7)$ \\
\hline 9 & $\mathrm{E}, \mathrm{DA}$ & 5 & - & $5(3.6)$ \\
\hline 10 & $\mathrm{P}, \mathrm{E}, \mathrm{FOX}$ & - & 1 & $1(0.7)$ \\
\hline 11 & $\mathrm{P}, \mathrm{FOX}, \mathrm{OX}$ & - & 1 & $1(0.7)$ \\
\hline 12 & P, E, STX & 1 & 1 & $2(1.4)$ \\
\hline 13 & $\mathrm{P}, \mathrm{E}, \mathrm{CIP}$ & 1 & - & $1(0.7)$ \\
\hline 14 & $\mathrm{P}, \mathrm{E}, \mathrm{DA}$ & 32 & 11 & $43(30.9)$ \\
\hline 15 & $\mathrm{P}, \mathrm{E}, \mathrm{CN}$ & 1 & - & $1(0.7)$ \\
\hline 16 & $\mathrm{P}, \mathrm{DA}, \mathrm{FOX}$ & - & 1 & $1(0.7)$ \\
\hline 17 & $\mathrm{P}, \mathrm{CIP}, \mathrm{RD}$ & 1 & - & $1(0.7)$ \\
\hline 18 & $\mathrm{P}, \mathrm{E}, \mathrm{DA}, \mathrm{FOX}$ & - & 3 & $3(2.2)$ \\
\hline 19 & $\mathrm{P}, \mathrm{E}, \mathrm{DA}, \mathrm{OX}$ & - & 7 & $7(5.0)$ \\
\hline 20 & P, E, DA, STX & 3 & 1 & $4(2.9)$ \\
\hline 21 & $\mathrm{P}, \mathrm{E}, \mathrm{DA}, \mathrm{CIP}$ & 2 & - & $2(1.4)$ \\
\hline 22 & $\mathrm{P}, \mathrm{E}, \mathrm{DA}, \mathrm{CN}$ & 2 & - & $2(1.4)$ \\
\hline 23 & $\mathrm{P}, \mathrm{E}, \mathrm{DA}, \mathrm{TE}$ & 1 & - & $1(0.7)$ \\
\hline 24 & $\mathrm{P}, \mathrm{E}, \mathrm{STX}, \mathrm{TE}$ & - & 1 & $1(0.7)$ \\
\hline 25 & $\mathrm{P}, \mathrm{E}, \mathrm{DA}, \mathrm{FOX}, \mathrm{OX}$ & - & 1 & $1(0.7)$ \\
\hline 26 & P, E, DA, STX, TGC & 1 & - & $1(0.7)$ \\
\hline 27 & P, E, DA, CN, TE & 1 & - & $1(0.7)$ \\
\hline 28 & P, E, DA, FOX, CIP & - & 1 & $1(0.7)$ \\
\hline 29 & $\mathrm{P}, \mathrm{E}, \mathrm{DA}, \mathrm{FOX}, \mathrm{CN}$ & - & 1 & $1(0.7)$ \\
\hline 30 & P, E, STX, CIP, TE & 1 & - & $1(0.7)$ \\
\hline 31 & $\mathrm{P}, \mathrm{E}, \mathrm{DA}, \mathrm{STX}, \mathrm{CN}$ & 1 & - & $1(0.7)$ \\
\hline 32 & $\mathrm{P}, \mathrm{E}, \mathrm{DA}, \mathrm{FOX}, \mathrm{OX}, \mathrm{CN}$ & - & 1 & $1(0.7)$ \\
\hline 33 & $\mathrm{P}, \mathrm{E}, \mathrm{DA}, \mathrm{FOX}, \mathrm{OX}, \mathrm{LZD}$ & - & 1 & $1(0.7)$ \\
\hline 34 & $\mathrm{P}, \mathrm{E}, \mathrm{DA}, \mathrm{FOX}, \mathrm{CIP}, \mathrm{CN}$ & - & 1 & $1(0.7)$ \\
\hline 35 & $\mathrm{P}, \mathrm{E}, \mathrm{DA}, \mathrm{FOX}, \mathrm{CN}, \mathrm{TE}$ & - & 1 & $1(0.7)$ \\
\hline 36 & $\mathrm{P}, \mathrm{E}, \mathrm{DA}, \mathrm{FOX}, \mathrm{CIP}, \mathrm{CN}, \mathrm{TE}$ & - & 1 & $1(0.7)$ \\
\hline 37 & P, E, DA, FOX, OX, CIP, CLO & - & 1 & $1(0.7)$ \\
\hline 38 & P, E, DA, STX, CN, CIP, CLO, TE & 1 & - & $1(0.7)$ \\
\hline
\end{tabular}

*Six isolates were susceptible to all antimicrobial tested. P: penicillin (10 U); FOX: cefoxitin (30 $\mu \mathrm{g})$; CLO: chloramphenicol (30 $\mu \mathrm{g})$; CIP: ciprofloxacin (5 $\mu \mathrm{g})$; DA: clindamycin $(2 \mu \mathrm{g})$; E: erythromycin $(15 \mu \mathrm{g})$; CN: gentamicin $(10 \mu \mathrm{g})$; LZD: linezolid (30 $\mu \mathrm{g}) ; \mathrm{RD}$ : rifampicin $(5 \mu \mathrm{g})$ STX: sulfamethoxazole/trimethoprim $(23.75 / 1.25 \mu \mathrm{g})$; TE: tetracycline $(30 \mu \mathrm{g})$; TGC: tigecycline $(15 \mu \mathrm{g})$. - : not detected.

continuous monitoring of the antimicrobial susceptibility and virulence potential of $S$. aureus for the proper management of the infections caused by this bacterium.

TSST- 1 is a potent exotoxin of the superantigens family that activates $\mathrm{T}$ lymphocytes, resulting in systemic inflammation and shock due to the overproduction of cytokines [55]. Infections caused by tst-positive $S$. aureus are also potentially fatal [53] and remain an important cause of morbidity among young people, especially in young women [50]. Although tst gene is located in a pathogenicity island, it can also be transferred among different isolates by a helper bacteriophage [56].

The limitation of this analysis is that the presence of virulence-encoding genes was evaluated only by PCR, which does not reflect the expression of the phenotype. However, nasal colonization by $S$. aureus harboring PVL and TSST-1 encoding genes in healthcare workers and students attending the hospital settings may represent a potential source for the emergence of a successful $S$. aureus strain highly adapted in hospital environment due to the silent spread of these virulence markers to multidrug-resistant strains and hence severe and difficult to treat infection in patients. Moreover, both populations may serve as agents of cross-transmission of this strain between the hospital and community individuals.

3.5. MRSA Isolates Harboring Different SCCmec and REPPCR Types Are Grouped into Four Main Clusters. MRSA isolates were distributed into six SCCmec types and the type I 


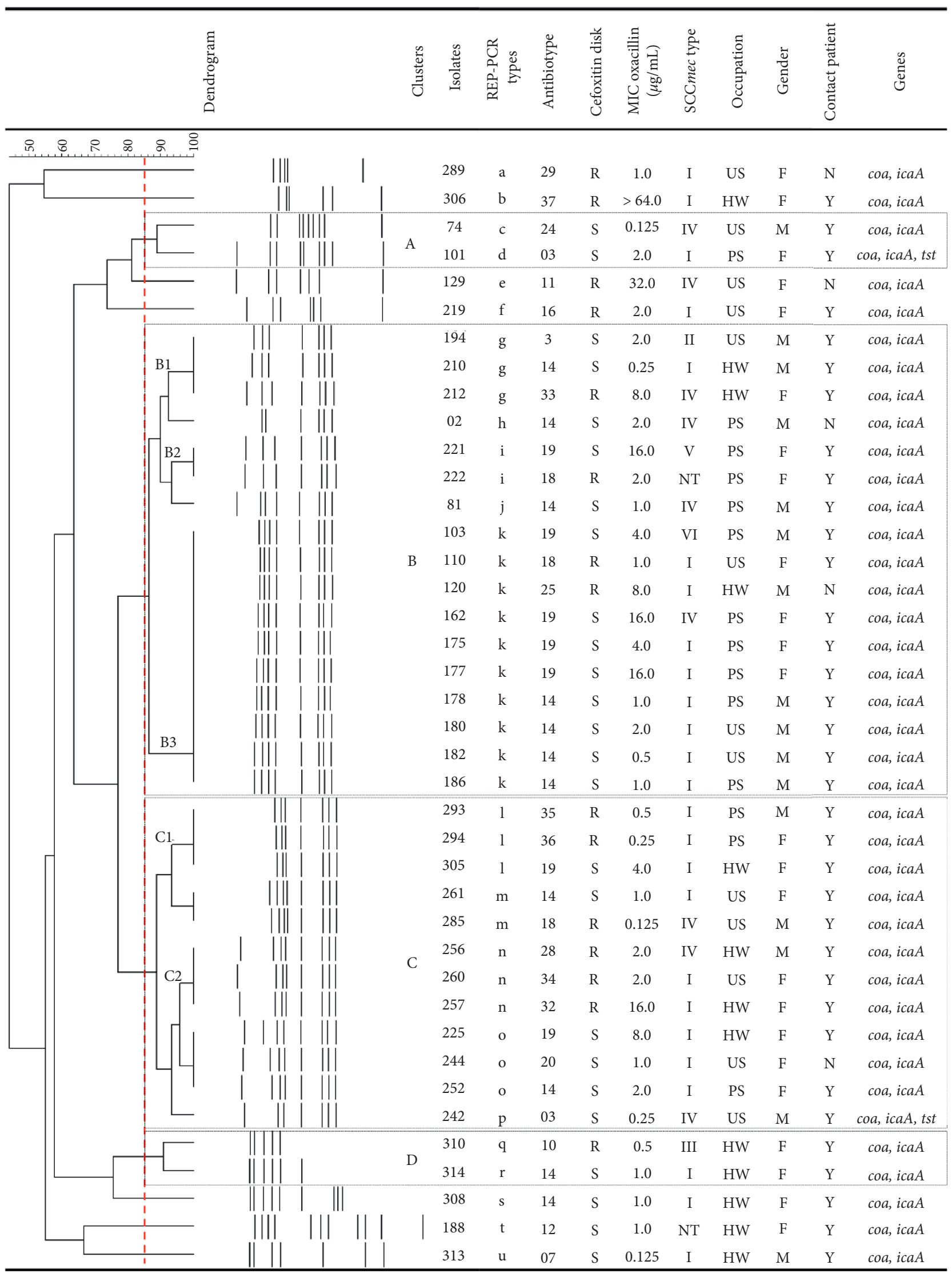

FIGURE 2: Phenotypic and molecular characteristics of 40 methicillin-resistant Staphylococcus aureus (MRSA, mecA positive) and descriptive variables of MRSA carriers. The UPGMA banding pattern obtained by REP-PCR based on Dice similarity coefficient showing the genetic relatedness of MRSAs. Vertical dashed line marks the position of similarity coefficient value of 0.85 . REP-PCR: repetitive element sequence based-polymerase chain reaction; MIC: minimal inhibitory concentration; R resistant; S susceptible; SCCmec: staphylococcal cassette chromosome mec; NT: nontypeable; HW: healthcare worker; US: undergraduate student; PS: postgraduate student; F: female; M: male; N: no; Y: yes. 
TABLE 5: Distribution of virulence encoding genes profiles in methicillin-susceptible and methicillin-resistant Staphylococcus aureus isolated from the anterior nares of healthcare workers and students attending the University Hospital of Londrina in southern Brazil.

\begin{tabular}{lccc}
\hline Virulence marker & Number of isolates & MRSA $n=40$ & Total $N=139(\%)$ \\
\hline coa, $i c a A$ & 71 & 38 & $109(78.4)$ \\
coa, $t s t$ & 3 & - & $3(2.2)$ \\
coa, $i c a A$, tst & 16 & 2 & $18(12.9)$ \\
coa, $i c a A, l u k S-P V / l u k F-P V$ & 8 & - & $8(5.8)$ \\
coa, $i c a A$, st, lukS-PV/lukF-PV & 1 & - & $1(0.7)$ \\
\hline
\end{tabular}

coa: coagulase; icaA: intercellular adhesion locus encoding N-acetylglucosaminyltransferase; tst: toxic shock syndrome toxin; lukS-PV and lukF-PV: $\beta$-poreforming Panton-Valentine leukocidin. - : not detected.

$(25 / 40,62.5 \%)$ was the most frequent, followed by type IV $(9 / 40,22.5 \%)$. One isolate each $(1 / 40,2.5 \%)$ harbored the SCCmec types II, III, V, and VI; two isolates (2/40, 5.0\%) were classified as NT (Figure 2). The SCCmec types identified herein were also detected in MRSA isolated from different staphylococcal infections in our hospital, where SCCmec type II was the most prevalent $[3,6,7]$.

Visual observation of bands generated by REP-PCR typing showed a total of 21 banding patterns (named a to $\mathrm{u}$ ), indicating a relatively high genetic diversity among MRSA isolates. REP-PCR banding patterns were automatically sized and all isolates with the same type were recognized to be identical. The level of similarity between the REP-PCR fingerprinting of the MRSA isolates ranged from 44.1 to $100 \%$. Clusters analysis, by using a cutoff value of $85 \%$ [3], revealed that the majority of MRSA isolates were distributed into four groups (named as A to $\mathrm{D}$ clusters). Seven isolates exhibited unique fingerprinting patterns. Clusters A and D harbored two isolates each of the different REP-PCR and SCCmec types. Cluster B consisted of 17 isolates, comprising five REP-PCR ( $g, h, i, j$, and k) and six SCCmec (I, II, IV, V, VI, and NT) different types, which were grouped in three subclusters (B1, B2, and B3). Finally, cluster $\mathrm{C}$ consisted of 12 isolates, comprising five REP-PCR (l, m, n, o, and p) and two SCCmec (I and IV) different types, which were grouped in two subclusters (C1 and C2) (Figure 2).

OS-MRSA isolates harbored the SCCmec type I (12/18, $66.7 \%)$, type IV $(4 / 18,22.2 \%)$, and type II $(1 / 18,5.6 \%)$ and one was NT $(1 / 18,5.6 \%)$. Moreover, most OS-MRSA isolates belonged to clusters $\mathrm{B}(8 / 18,44.4 \%)$ and $\mathrm{C}(4 / 18,22.2 \%)$. These results may explain the high prevalence of OS-MRSA nasal carriers in our study.

\section{Conclusion}

This study provided an overview of $S$. aureus nasal carriage among healthcare workers and students attending our hospital. We observed a high prevalence of $S$. aureus exhibiting different antimicrobial resistance profiles, including multidrug-resistant and OS-MRSA isolates; higher prevalence of MRSA harboring the SCCmec types I and IV, which is in contrast to our previous studies reporting the prevalence of SCCmec type II among MRSA isolated from different infections $[3,6]$; and the presence of PVL and TSST-1 encoding genes among these isolates. These data alert us for continuous surveillance of potential reservoirs of $S$. aureus and improvement in the infections control measures for detecting the various methicillin susceptibility phenotypes and virulence potential of $S$. aureus.

\section{Data Availability}

The data generated and analyzed in the current study are included in the article. Data are also available from the corresponding author upon reasonable request.

\section{Conflicts of Interest}

The authors declare no conflicts of interest.

\section{Acknowledgments}

This study was supported by grants from Coordenação de Aperfeiçoamento de Pessoal de Nível Superior (CAPES, Financial Code 01). T. D., F. C. D., G. B. G., and C. F. de O. were funded by a graduate scholarship from CAPES. E. R. T. was funded by a research fellowship from CAPES. L. M. Y. and S. F. Y.-O. were funded by a fellowship from Conselho Nacional de Desenvolvimento Científico e Tecnológico (CNPq).

\section{References}

[1] B. Krismer, C. Weidenmaier, A. Zipperer, and A. Peschel, "The commensal lifestyle of Staphylococcus aureus and its interactions with the nasal microbiota," Nature Reviews Microbiology, vol. 15, no. 11, pp. 675-687, 2017.

[2] F. D. Lowy, "Staphylococcus aureus infections," New England Journal of Medicine, vol. 339, no. 8, pp. 520-532, 1998.

[3] C. F. d. Oliveira, A. T. Morey, J. P. Santos et al., "Molecular and phenotypic characteristics of methicillin-resistant Staphylococcus aureus isolated from hospitalized patients," The Journal of Infection in Developing Countries, vol. 9, no. 07, pp. 743-751, 2015.

[4] A. S. Lee, H. de Lencastre, J. Garau et al., "Methicillin-resistant Staphylococcus aureus," Nature Reviews Disease Primers, vol. 4, pp. 1-23, 2018.

[5] F. C. Duarte, T. Danelli, M. A. G. Ribeiro et al., "Bacteremia causada por Staphylococcus aureus: Uma análise de quinze anos da sensibilidade a antimicrobianos em um hospital terciário do Brasil," Revista de Epidemiologia e Controle de Infecção, vol. 8, no. 3, pp. 232-238, 2018.

[6] F. C. Duarte, E. R. Tavares, T. Danelli et al., "Disseminated Clonal Complex 5 (CC5) methicillin-resistant Staphylococcus aureus SCCmec type II in a tertiary hospital of Southern 
Brazil," Revista do Instituto de Medicina Tropical de São Paulo, vol. 60, p. e32, 2018.

[7] F. C. Duarte, T. Danelli, E. R. Tavares et al., "Fatal sepsis caused by mecA-positive oxacillin-susceptible Staphylococcus aureus: first report in a tertiary hospital of southern Brazil," Journal of Infection and Chemotherapy, vol. 25, no. 4, pp. 293-297, 2019.

[8] B. C. Young, C.-H. Wu, N. C. Gordon et al., "Severe infections emerge from commensal bacteria by adaptive evolution," Elife, vol. 6, p. e30637, 2017.

[9] F. P. Paling, M. Wolkewitz, L. G. M. Bode et al., "Staphylococcus aureus colonization at ICU admission as a risk factor for developing S. aureus ICU pneumonia," Clinical Microbiololy and Infection, vol. 23, pp. 49.e9-49.e14, 2017.

[10] A. Maayan-Metzger, T. Strauss, C. Rubin et al., "Clinical evaluation of early acquisition of Staphylococcus aureus carriage by newborns," International Journal of Infectious Diseases, vol. 64, pp. 9-14, 2017.

[11] H. F. L. Wertheim, M. v. Kleef, M. C. Vos, A. Ott, H. A. Verbrugh, and W. Fokkens, "Nose picking and nasal carriage of Staphylococcus aureus," Infection Control \& Hospital Epidemiology, vol. 27, no. 08, pp. 863-867, 2006.

[12] L. Mody, L. L. Washer, K. S. Kaye et al., "Multidrug-resistant organisms in hospitals: what is on patient hands and in their rooms?" Clinical Infectious Diseases, vol. 69, no. 11, pp. 1837-1844, 2019.

[13] E. C. B. F. d. Silva, M. d. G. C. Antas, A. M. B. Neto, M. A. Rabelo, F. L. d. Melo, and M. A. V. Maciel, "Prevalence and risk factors for Staphylococcus aureus in healthcare workers at a University Hospital of Recife-PE," Brazilian Journal of Infectious Diseases, vol. 12, no. 6, pp. 504-508, 2008.

[14] B. Hogan, R. Rakotozandrindrainy, H. Al-Emran et al., "Prevalence of nasal colonisation by methicillin-sensitive and methicillin-resistant Staphylococcus aureus among healthcare workers and students in Madagascar," BMC Infectious Diseases, vol. 16, no. 1, p. 420, 2016.

[15] N. R. Hussein, M. S. Assafi, and T. Ijaz, "Methicillin-resistant Staphylococcus aureus nasal colonisation amongst healthcare workers in Kurdistan Region, Iraq," Journal of Global Antimicrobial Resistance, vol. 9, pp. 78-81, 2017.

[16] M. Emaneini, F. Jabalameli, H. Rahdar, W. B. V. Leeuwen, and R. Beigverdi, "Nasal carriage rate of methicillin resistant Staphylococcus aureus among Iranian healthcare workers: a systematic review and meta-analysis," Revista da Sociedade Brasileira de Medicina Tropical, vol. 50, no. 5, pp. 590-597, 2017.

[17] H. Legese, A. G. Kahsay, A. Kahsay et al., "Nasal carriage, risk factors and antimicrobial susceptibility pattern of methicillin resistant Staphylococcus aureus among healthcare workers in Adigrat and Wukro hospitals, Tigray, Northern Ethiopia," BMC Research Notes, vol. 11, no. 1, pp. 1-6, 2018.

[18] T. S. El-Mahdy, M. H. Al-Agamy, M. Emara M et al., "Complex clonal diversity of Staphylococcus aureus nasal colonization among community personnel, healthcare workers, and clinical students in the Eastern Province, Saudi Arabia," BioMed Research International, vol. 2018, Article ID 4208762, 2018.

[19] T. Conceição, H. de Lencastre, and M. Aires-de-Sousa, "Carriage of Staphylococcus aureus among Portuguese nursing students: a longitudinal cohort study over four years of education," PLoS One, vol. 12, no. 11, p. e0188855, 2017.

[20] K. A. Prates, A. M. Torres, L. B. Garcia, S. F. Y. Ogatta, C. L. Cardoso, and M. C. B. Tognim, "Nasal carriage of methicillin-resistant Staphylococcus aureus in university students," Brazilian Journal of Infectious Diseases, vol. 14, no. 3, pp. 316-318, 2010.

[21] K. Becker, R. L. Skov, and C. Von Eiff, "Staphylococcus, Micrococcus, and other catalase-positive cocci," in Manual of Clinical Microbiology, J. H. Jorgensen and M. A. Pfaller, Eds., p. 2726, ASM, Washington, DC, 2017.

[22] S. Hirotaki, T. Sasaki, K. Kuwahara-Arai, and K. Hiramatsu, "Rapid and accurate identification of human-associated Staphylococci by use of multiplex PCR," Journal of Clinical Microbiology, vol. 49, no. 10, pp. 3627-3631, 2011.

[23] Clinical and Laboratory Standards Institute (CLSI), Performance Standards for Antimicrobial Susceptibility Testing, Clinical and Laboratory Standard Institute, Wayne, PA, 29 edition, 2019.

[24] EUCAST. European Committee on Antimicrobial Susceptibility Testing, http://www.eucast.org.

[25] R. Khatib, K. Riederer, M. Sharma, S. Shemes, S. P. Iyer, and S. Szpunar, "Screening for intermediately vancomycin-susceptible and vancomycin-heteroresistant Staphylococcus aureus by use of vancomycin-supplemented brain heart infusion agar biplates: defining growth interpretation criteria based on gold standard confirmation," Journal of Clinical Microbiology, vol. 53, no. 11, pp. 3543-3546, 2015.

[26] F. M. Ausubel, R. Brent, R. E. Kingston et al., Short Protocols in Molecular Biology, John Wiley \& Sons, New York, NY, USA, 1999.

[27] C. Milheiriço, D. C. Oliveira, and H. de Lencastre, "Update to the multiplex PCR strategy for assignment of mec element types in Staphylococcus aureus," Antimicrobial Agents and Chemotherapy, vol. 51, no. 9, pp. 3374-3377, 2007.

[28] S. J. Campbell, H. S. Deshmukh, C. L. Nelson et al., "Genotypic characteristics of Staphylococcus aureus isolates from a multinational trial of complicated skin and skin structure infections," Journal of Clinical Microbiology, vol. 46, no. 2, pp. 678-684, 2008.

[29] H. K. Tiwari, D. Sapkota, and M. R. Sen, "Detection of Staphylococcus aureus using coagulase (Coa) gene PCR as the gold standard," Nepal Medical College Journal, vol. 10, no. 2, pp. 129-131, 2008.

[30] V. G. Del Vecchio, J. M. Petroziello, M. J. Gress et al., "Molecular genotyping of methicillin-resistant Staphylococcus aureus via fluorophore-enhanced repetitive-sequence PCR," Journal of Clinical Microbiology, vol. 33, no. 8, pp. 2141-2144, 1995.

[31] A. van der Zee, H. Verbakel, J.-C. van Zon et al., "Molecular genotyping of Staphylococcus aureus strains: comparison of repetitive element sequence-based PCR with various typing methods and isolation of a novel epidemicity marker," Journal of Clinical Microbiology, vol. 37, no. 2, pp. 342-349, 1999.

[32] P. H. A. Sneath and R. Sokal, Numerical Taxonomy: The Principles and Practices of Numerical Classifications, W.H. Freem., San Francisco. CA. USA, 1973.

[33] A. van Belkum, N. J. Verkaik, C. P. de Vogel et al., "Reclassification of Staphylococcus aureus nasal carriage types," The Journal of Infectious Diseases, vol. 199, no. 12, pp. 1820-1826, 2009.

[34] J. Legrand, L. Temime, C. Lawrence, J. L. Herrmann, P. Y. Boelle, and D. Guillemot, "Occupational determinants of methicillin-resistant Staphylococcus aureus colonization among healthcare workers: a longitudinal study in a rehabilitation center," Infection Control \& Hospital Epidemiology, vol. 36, no. 7, pp. 767-776, 2015.

[35] P. E. Budri, A. C. Shore, D. C. Coleman et al., "Observational cross-sectional study of nasal staphylococcal species of 
medical students of diverse geographical origin, prior to healthcare exposure: prevalence of SCCmec, fusC, fusB and the arginine catabolite mobile element (ACME) in the absence of selective antibiotic pressure," BMJ Open, vol. 8, no. 4, p. e020391, 2018.

[36] X. Xie, X. Dai, L. Ni et al., "Molecular epidemiology and virulence characteristics of Staphylococcus aureus nasal colonization in medical laboratory staff: comparison between microbiological and non-microbiological laboratories," $B M C$ Infectious Diseases, vol. 18, no. 1, p. 122, 2018.

[37] M. Dulon, C. Peters, A. Schablon et al., "MRSA carriage among healthcare workers in non-outbreak settings in Europe and the United States: a systematic review," BMC Infectious Diseases, vol. 14, p. 363, 2014.

[38] N. Pourramezan, S. Ohadian Moghadam, and M. R. Pourmand, "Methicillin-resistant Staphylococcus aureus tracking spread among health-care workers and hospitalized patients in critical wards at a university hospital, Tehran, Iran," New Microbes and New Infections, vol. 27, pp. 29-35, 2019.

[39] A. Bettin, C. Causil, and N. Reyes, "Molecular identification and antimicrobial susceptibility of Staphylococcus aureus nasal isolates from medical students in Cartagena, Colombia," The Brazilian Journal of Infectious Diseases, vol. 16, no. 4, pp. 329-334, 2012.

[40] M. De Giusti, L. Marinelli, C. Aurigemma et al., "Prevalence of Staphylococcus aureus colonization and antibiotic susceptibility: a survey among biomedical students," Public Health, vol. 127, no. 4, pp. 392-394, 2013.

[41] M. S. M. Carvalho, D. F. R. d. Andrade, Á. F. L. d. Sousa et al., "Colonização nasal por Staphylococcus aureus entre estudantes de Enfermagem: subsídios para monitorização," Revista Brasileira de Enfermagem, vol. 69, no. 6, pp. 10461051, 2016.

[42] A.-P. Magiorakos, A. Srinivasan, R. B. Carey et al., "Multidrug-resistant, extensively drug-resistant and pandrug-resistant bacteria: an international expert proposal for interim standard definitions for acquired resistance," Clinical Microbiology and Infection, vol. 18, no. 3, pp. 268-281, 2012.

[43] M. Andrade-Figueiredo and T. C. Leal-Balbino, "Clonal diversity and epidemiological characteristics of Staphylococcus aureus: high prevalence of oxacillin-susceptible mecA-positive Staphylococcus aureus (OS-MRSA) associated with clinical isolates in Brazil," BMC Microbiology, vol. 16, no. 1, p. 115, 2016.

[44] M. K. Proulx, S. G. Palace, S. Gandra et al., "Reversion from methicillin susceptibility to methicillin resistance in Staphylococcus aureus during treatment of bacteremia," Journal of Infectious Diseases, vol. 213, no. 6, pp. 1041-1048, 2016.

[45] K. Tam and V. J. Torres, "Staphylococcus aureus secreted toxins and extracellular enzymes," Microbiology Spectrum, vol. 7, no. 2, 2019.

[46] R. W. Fairbrother, "Coagulase production as a criterion for the classification of the Staphylococci," The Journal of $\mathrm{Pa}$ thology and Bacteriology, vol. 50, no. 1, pp. 83-88, 1940.

[47] S. E. Cramton, C. Gerke, N. F. Schnell, W. W. Nichols, and F. Götz, "The intercellular adhesion (ica) locus is present in Staphylococcus aureus and is required for biofilm formation," Infection and Immunity, vol. 67, no. 10, pp. 5427-5433, 1999.

[48] A. Kropec, T. Maira-Litran, K. K. Jefferson et al., "Poly-Nacetylglucosamine production in Staphylococcus aureus is essential for virulence in murine models of systemic infection," Infection and Immunity, vol. 73, no. 10, pp. 6868-6876, 2005.
[49] K. Saeed, I. Gould, S. Esposito et al., "Panton-valentine leukocidin-positive Staphylococcus aureus: a position statement from the international society of chemotherapy," International Journal of Antimicrobial Agents, vol. 51, no. 1, pp. 16-25, 2018.

[50] A. S. DeVries, L. Lesher, P. M. Schlievert et al., "Staphylococcal toxic shock syndrome 2000-2006: epidemiology, clinical features, and molecular characteristics," PLoS One, vol. 6, no. 8, pp. 1-8, 2011.

[51] J. Kaneko and Y. Kamio, "Bacterial two-component and hetero-heptameric pore-forming cytolytic toxins: structures, pore-forming mechanism, and organization of the genes," Bioscience, Biotechnology, and Biochemistry, vol. 68, no. 5, pp. 981-1003, 2004.

[52] N. Sicot, N. Khanafer, V. Meyssonnier et al., "Methicillin resistance is not a predictor of severity in community-acquired Staphylococcus aureus necrotizing pneumonia-results of a prospective observational study," Clinical Microbiology and Infection, vol. 19, no. 3, pp. E142-E148, 2013.

[53] Y. Gillet, T. Henry, and F. Vandenesch, "Fulminant staphylococcal infections," Microbiology Spectrum, vol. 6, no. 5, 2018.

[54] O. Dumitrescu, P. Choudhury, S. Boisset et al., “ $\beta$-Lactams interfering with PBP1 induce panton-valentine leukocidin expression by triggering sarA and rot global regulators of Staphylococcus aureus," Antimicrobial Agents and Chemotherapy, vol. 55, no. 7, pp. 3261-3271, 2011.

[55] S. X. Xu and J. K. McCormick, "Staphylococcal superantigens in colonization and disease," Frontiers in Cellular and Infection Microbiology, vol. 2, pp. 1-11, 2012.

[56] R. P. Novick and G. Ram, "Staphylococcal pathogenicity islands-movers and shakers in the genomic firmament," Current Opinion in Microbiology, vol. 38, pp. 197-204, 2017. 